\title{
Does the Milky Way produce a starburst wind?
}

\section{Brian A. Keeney, Charles W. Danforth, John T. Stocke, Steven V. Penton and J. Michael Shull}

Center for Astrophysics and Space Astronomy, Department of Astrophysical and Planetary Sciences, University of Colorado, Box 389, Boulder, CO 80309, USA

email: keeney@colorado.edu

\begin{abstract}
We present new FUSE and HST/STIS observations of two high Galactic latitude AGN that we use to measure the nuclear wind velocity of the Milky Way for the first time. We find high velocity C III \& O VI at a heliocentric velocity of $+163 \pm 8 \mathrm{~km} \mathrm{~s}^{-1}$ and C III \& N V at $-105 \pm 14 \mathrm{~km} \mathrm{~s}^{-1}$ toward PKS 2005-489 $\left(l=350^{\circ}, b=-33^{\circ}\right)$ and C III, C IV, \& Si IV and C III \& O VI toward Mrk $1383\left(l=349^{\circ}, b=55^{\circ}\right)$ at $+48 \pm 4 \mathrm{~km} \mathrm{~s}^{-1}$ and $+94 \pm 7 \mathrm{~km} \mathrm{~s}^{-1}$, respectively. We have estimated the Galactocentric escape velocity of gas above the Galactic Centre along the PKS 2005-489 and Mrk 1383 sight-lines to be $\geqslant 215 \pm 35$ and $\geqslant 145 \pm 25 \mathrm{~km} \mathrm{~s}^{-1}$, respectively. Thus, we have detected both infalling and bound outflowing gas from the Galactic Centre, indicating that the Milky Way does not produce a starburst wind, but a bound Galactic "fountain" instead.
\end{abstract}

\section{Introduction}

Starburst-driven superwinds have long been suggested as a mechanism by which metals and energy can be transported from galaxies to enrich their surroundings. Superwinds have been studied around nearby galaxies in X-ray and $\mathrm{H} \alpha$ emission and in $\mathrm{UV}$ and optical absorption using the stellar continuum and nearby AGN as background sources (e.g. Martin 1999; Heckman et al. 2000, 2001; Martin et al. 2002; Keeney et al. 2005). Absorption line studies of nearby starburst galaxies typically detect outflow velocities of 400-1000 $\mathrm{km} \mathrm{s}^{-1}$; however, they suffer from an ambiguity in the distance between the background source and the absorbing gas and so cannot prove that starburst galaxies commonly generate winds capable of escaping the galaxy's gravitational potential.

Despite success detecting winds in nearby starburst galaxies, no attempts have been made to study the wind emanating from the starburst at the centre of our own Galaxy. We present new FUSE, and HST/STIS G140M and E140M observations of two high Galactic latitude AGN that lie in the direction of the Galactic Centre (PKS 2005-489 at $l=350^{\circ}, b=-33^{\circ}$ and Mrk 1383 at $l=349^{\circ}, b=55^{\circ}$ ), which we use to detect and measure the Milky Way nuclear wind velocity for the first time. In addition to the two extragalactic sight-lines, we have data on four comparison B stars (HD 121968 \& HD 125924 toward Mrk 1383 and HD 187311 \& HD 191466 toward PKS 2005-489) that were used to remove foreground contaminating components not associated with the Galactic Centre (e.g. the Sco-Cen OB associations).

\section{Results}

Our spectra of PKS 2005-489 and its comparison stars allow us to examine the absorption line profiles of C III $\lambda 977$, O VI $\lambda \lambda 1032,1038$ and N V $\lambda \lambda 1238,1242$. The Mrk 1383 data cover a larger wavelength range, allowing us to examine additionally the profiles of 

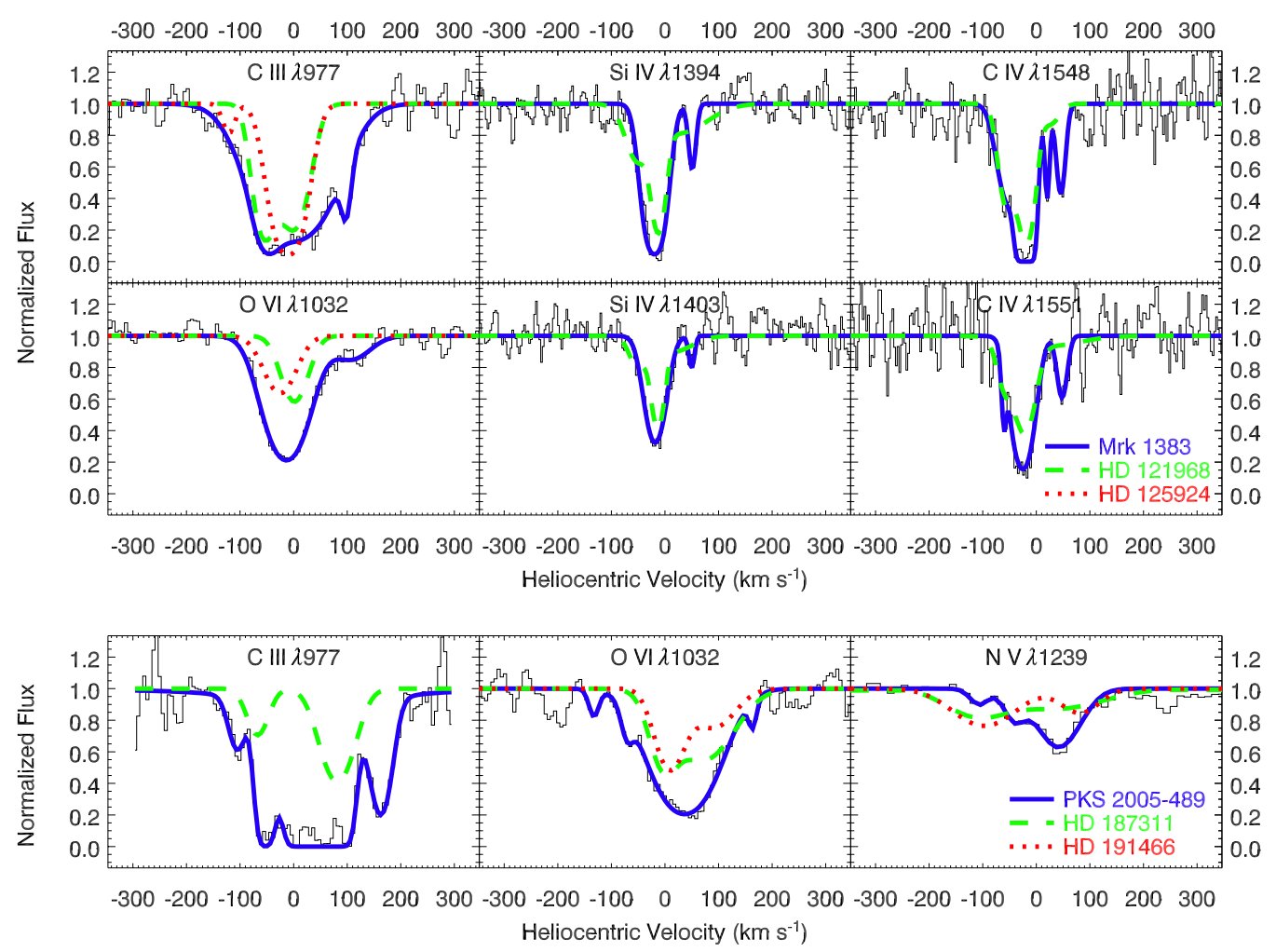

Figure 1. FUSE and HST/STIS spectra of Mrk 1383 (top) and PKS 2005-489 (bottom). Best-fit Voigt profiles for the AGN spectra are shown as solid lines and best-fit Voigt profiles for the comparison star spectra (data not shown) are shown as dashed and dotted lines.

Si IV $\lambda \lambda 1394,1403$ and C IV $\lambda \lambda 1548,1551$. Unfortunately, O VI $\lambda 1038$ and N V $\lambda 1242$ are not usable because O VI $\lambda 1038$ is severely blended with $\mathrm{C}$ II* and $\mathrm{H}_{2}$ lines in all of the comparison star spectra and N V $\lambda 1242$ is not detected in the AGN spectra.

Multi-component Voigt profiles were fit to the high ionisation lines in each spectrum and all interstellar velocity components in the comparison star spectra were subtracted from the AGN data. Fig. 1 shows the high ionisation absorption lines in the AGN spectra, with best-fit Voigt profiles overlaid. The best-fit profiles for the comparison star spectra are also shown.

Fig. 2 shows the best-fit Voigt profiles for the foreground-subtracted AGN spectra. We find high velocity C III \& O VI at a heliocentric velocity of $+163 \pm 8 \mathrm{~km} \mathrm{~s}^{-1}$ and C III \& $\mathrm{N} \mathrm{V}$ at $-105 \pm 14 \mathrm{~km} \mathrm{~s}^{-1}$ toward PKS 2005-489. The latter component is bracketed by two O VI velocity components, perhaps representing the collisionally ionised interface of an O VI high velocity cloud (see e.g. Sembach et al. 2003, 2004; Collins et al. 2003, 2004). Toward Mrk 1383 we find high velocity C III, C IV \& Si IV at a heliocentric velocity of $+48 \pm 4 \mathrm{~km} \mathrm{~s}^{-1}$ and C III \& O VI at $+94 \pm 7 \mathrm{~km} \mathrm{~s}^{-1}$.

To determine whether these high velocity components are bound to the Galaxy, we have estimated the escape velocity at Galactocentric radii of 5.7 and $12.5 \mathrm{kpc}$, the radii of the absorbing gas toward PKS 2005-489 and Mrk 1383 respectively, if this gas is located directly above the Galactic Centre. Assuming a Keplerian gravitational potential with a mass of $\geqslant(3 \pm 1) \times 10^{10} M_{\odot}$ (the mass of the Galactic Bulge; Blum 1995) yields Galactocentric escape velocities toward PKS 2005-489 and Mrk 1383 of $\geqslant 215 \pm 35$ and 


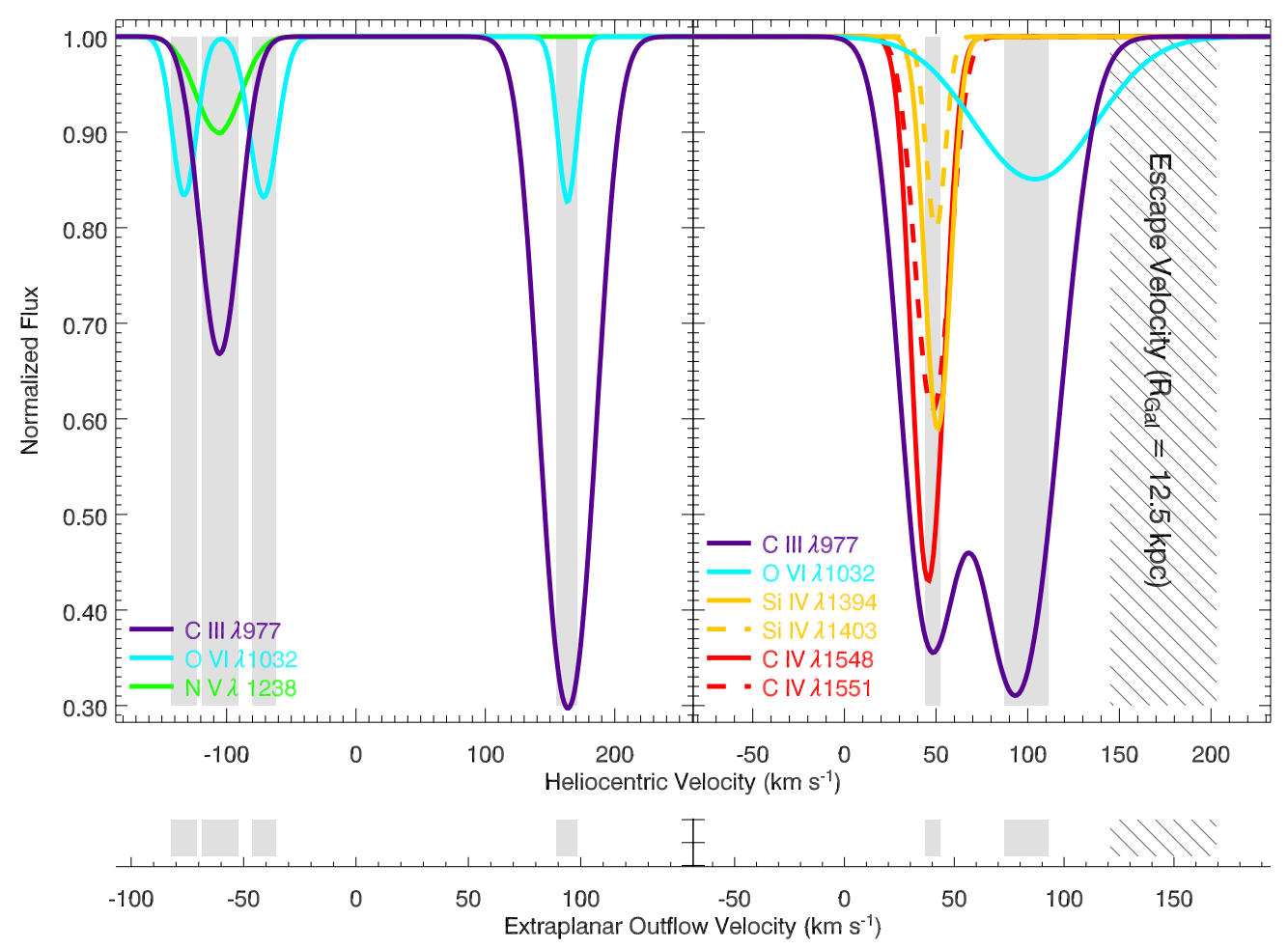

Figure 2. High velocity absorption components detected toward PKS 2005-489 (left) and Mrk 1383 (right). The solid grey bars indicate the $1 \sigma$ velocity range for each of the high velocity components, and the hatched region indicates the $1 \sigma$ velocity range for the escape velocity of gas above the Galactic Centre along the Mrk 1383 sight-line (the extra-planar escape velocity along the PKS 2005-489 sight-line is $\geqslant 215 \pm 35 \mathrm{~km} \mathrm{~s}^{-1}$ ).

$\geqslant 144 \pm 24 \mathrm{~km} \mathrm{~s}^{-1}$ respectively. Our estimate of the escape velocity along the Mrk 1383 sight-line is shown relative to the absorption line velocities in Fig. 2; the escape velocity along the PKS 2005-489 sight-line lies well outside the displayed range of velocities.

We have detected both infalling and bound outflowing gas from the Galactic Centre. The absence of unbound gas favours a Galactic fountain model for our Galaxy rather than a starburst wind. Perhaps a shallower gravitational potential is required for unbound winds to form.

\section{References}

Blum, R. D., 1995, ApJ, 444, L89

Collins, J. A., Shull, J. M., Giroux, M. L., 2003, ApJ, 585, 336

Collins, J. A., Shull, J. M., Giroux, M. L., 2004, ApJ, 605, 216

Heckman, T. M., et al., 2000, ApJS, 129, 493

Heckman, T. M., et al., 2001, ApJ, 554, 1021

Keeney, B. A. et al., 2005, ApJ, 622, 267

Martin, C. L., 1999, ApJ, 513, 156

Martin, C. L., Kobulnicky, H. A., Heckman, T. M., 2002, ApJ, 574, 663

Sembach, K. R., et al., 2003, ApJS, 146, 165

Sembach, K. R., et al., 2004, ApJS, 150, 387 